\title{
Considering Comorbidity in Adolescents with Social Anxiety Disorder
}

\author{
Luis-Joaquin Garcia-Lopez ${ }^{\bowtie}$, Natalia Bonilla, and Jose-Antonio Muela-Martinez \\ Department of Psychology, Division of Clinical Psychology, University of Jaen, Jaen, Spain
}

\begin{abstract}
Social anxiety disorder is a highly prevalent psychiatric disorder, with elevated comorbidity rates with other mental health disorders and may cause severe negative consequences. In adolescence, there is a lack of research on how comorbid disorders to social anxiety tends to form particular associations. With a large sample of adolescents with a clinical diagnosis of social anxiety disorder, data have revealed that certain disorders are more frequent and tend to dwell on concrete aggregates. Thus, it may be particularly useful and efficient for mental health providers, pediatricians and school counselors to screen for generalized anxiety disorder and specific phobia when assessing $\mathrm{SAD}$ in youth. Overall, findings stress the presence of comorbidity being the rule rather than the exception in adolescents with social anxiety disorder, and the need for further examination of its impact on assessment and differential diagnosis on this psychiatric disorder.
\end{abstract}

Psychiatry Investig 2016;13(5):574-576

Key Words Adolescence, Assessment, Comorbidity, Social anxiety disorder.

\section{INTRODUCTION}

Social anxiety disorder (SAD) is a marked fear or anxiety of social situations in which the person is exposed to possible scrutiny by others. ${ }^{1}$ Lifetime prevalence estimates usually range between 3 and $9 \%$ in adolescence. ${ }^{2}$ Psychiatric SAD tends to be a chronic, stable condition that severely disrupts long-term functioning, and cause substantially increased risks of depression, suicide attempts, substance abuse, severe social restrictions, early school leaving, lower educational attainment, and victimization. ${ }^{3}$ In adolescents, previous data reveal small effect size of gender differences in the nature of socially-provoking situations (i.e., boys and girls share the same social fears), although girls report a higher frequency of situations of social anxiety., ${ }^{4,5}$ SAD usually comorbid highly with other mental health disorders and indeed, is the rule rather than the exception in adolescence. ${ }^{3}$ However, to date, only one study has examined the comorbidity of SAD specifically in adolescents with SAD. ${ }^{6}$ With a small sample size of adolescents with a clin-

Received: November 13, 2015 Revised: December 22, 2015

Accepted: January 23, 2016 Available online: July 11, 2016

$\triangle$ Correspondence: Luis-Joaquin Garcia-Lopez, PhD

Department of Psychology, Division of Clinical Psychology, University of Jaen,

Building C5, Campus Lagunillas, Jean 23071, Spain

Tel: +34-953213214, Fax: +34-953211814

E-mail: ljgarcia@ujaen.es, ljgarlo@cop.es

(a) This is an Open Access article distributed under the terms of the Creative Commons Attribution Non-Commercial License (http://creativecommons.org/licenses/by$\mathrm{nc} / 3.0$ ) which permits unrestricted non-commercial use, distribution, and reproduction in any medium, provided the original work is properly cited. ical diagnosis of SAD ( $\mathrm{n}=17)$, authors found $\mathrm{SAD}$ co-occurred highly with mood, substance abuse and other anxiety disorders. However, when the sample size is reduced, even a limited number of detected cases in a particular disorder may inflate caseness, which precludes from any final conclusion. Therefore, there is a strong need of research to examine comorbidity in youth with larger sample sizes. This study is aimed to cover this gap.

\section{METHODS}

The clinical sample was composed of 424 Spanish-speaking adolescents with SAD as the primary disorder according to The Anxiety Disorders Interview Schedule for DSM-IV: Child and Parent Version (ADIS-IV-C/P, the most widely used interview to assess anxiety disorders in youth, with excellent psychometric properties. ${ }^{8}$ Among them, 409 were diagnosed with a clinical diagnosis of generalized social anxiety disorder. Based on DSM-5, adolescents would have met criteria for SAD. Participants were 160 (39.1\%) boys and 249 (60.9\%) were girls who aged from 12 to 17 years $(\mathrm{M}=15.45, \mathrm{SD}=1.30)$.

The study was approved by the School District and the University Research Ethics Committee in compliance with the Code of Ethics of the World Medical Association (Declaration of Helsinki) and the Charter of Fundamental Rights of the European Union. Adolescents were recruited from public and private schools, which were selected using a clustered, random sampling method from the school lists of the Department of 
Education so that all school districts were represented. The use of this method meant that the socioeconomic status and ethnic composition of the overall sample was representative of the community. Interviews were conducted by clinical psychology graduate students who were trained in a 12-hour workshop and supervised by a licensed clinical psychologist with more than 15 years of experience with the interview (first author). A Kappa value of 0.92 was found in the social anxiety section.

\section{RESULTS}

One third ( $\mathrm{n}=135)$ of SAD sample $(\mathrm{n}=409)$ exhibited any comorbid disorder. Out of sample with comorbidity, 92 (22.5\%) had one comorbid disorder, 27 (6.6\%) exhibited two comorbid disorders, 10 (2.4\%) presented with three comorbid disorders, and $6(1.5 \%)$ had four or more comorbid disorders. When adolescents were grouped based on the number of comorbid disorders within each cluster, the comorbidity rates (out of 92 participants) revealed a different pattern. Comorbidity rates for those with only one comorbid disorder were as follows: specific phobia (SP; 48.9\%), generalized anxiety disorder (GAD; 26.1\%), AD/HD (7.6\%; 1.1\% inattentive subtype, 2.2\% hyperactive-impulsive and $4.3 \%$ combined subtype), dysthimic disorder (DD; 5.4\%), agoraphobia without history of panic disorder (3.3\%), separation anxiety disorder (2.2\%), posttraumatic stress disorder (PTSD; 2.2\%), panic disorder (PD) with or without agoraphobia (1.1\%), and major depression (1.1\%). Data for two grouped comorbid disorders $(n=27)$ were as follows: SP+GAD (48.2\% out of 27$)$, SP+DD (7.4\%), $\mathrm{SP}+\mathrm{AD} / \mathrm{HD}$ (7.4\%), SP+OCD (7.4\%), SP+Agoraphobia without history of Panic Disorder (7.4\%), GAD+DD (7.4\%), $\mathrm{GAD}+\mathrm{AD} / \mathrm{HD}$ (7.4\%), SP+Major Depression (3.7\%), and GAD+Major Depression (3.7\%). Three comorbid disorders (out of 10 ) were grouped as: SP+GAD+PTSD (20\%), and 10\% for each one of the 8 remaining combinations: $S P+G A D+D D$, $\mathrm{SP}+\mathrm{GAD}+$ major depression, $\mathrm{SP}+\mathrm{GAD}+\mathrm{PD}$ with or without agoraphobia, $\mathrm{SP}+\mathrm{GAD}+\mathrm{AD} / \mathrm{HD}$ (inattention subtype), $\mathrm{SP}+$ $\mathrm{AD} / \mathrm{HD}$ (combined subtype)+DD, $\mathrm{SP}+\mathrm{OCD}+$ Major Depression, GAD+PTSD+DD, and GAD+PD with or without agoraphobia+major depression. Finally, one third of participants with four or more comorbid disorders exhibiting the following comorbidity association: specific phobia+GAD+PTSD+ major depression. The remaining combinations only retained one subject per cluster. Figure 1 displays the percentage of

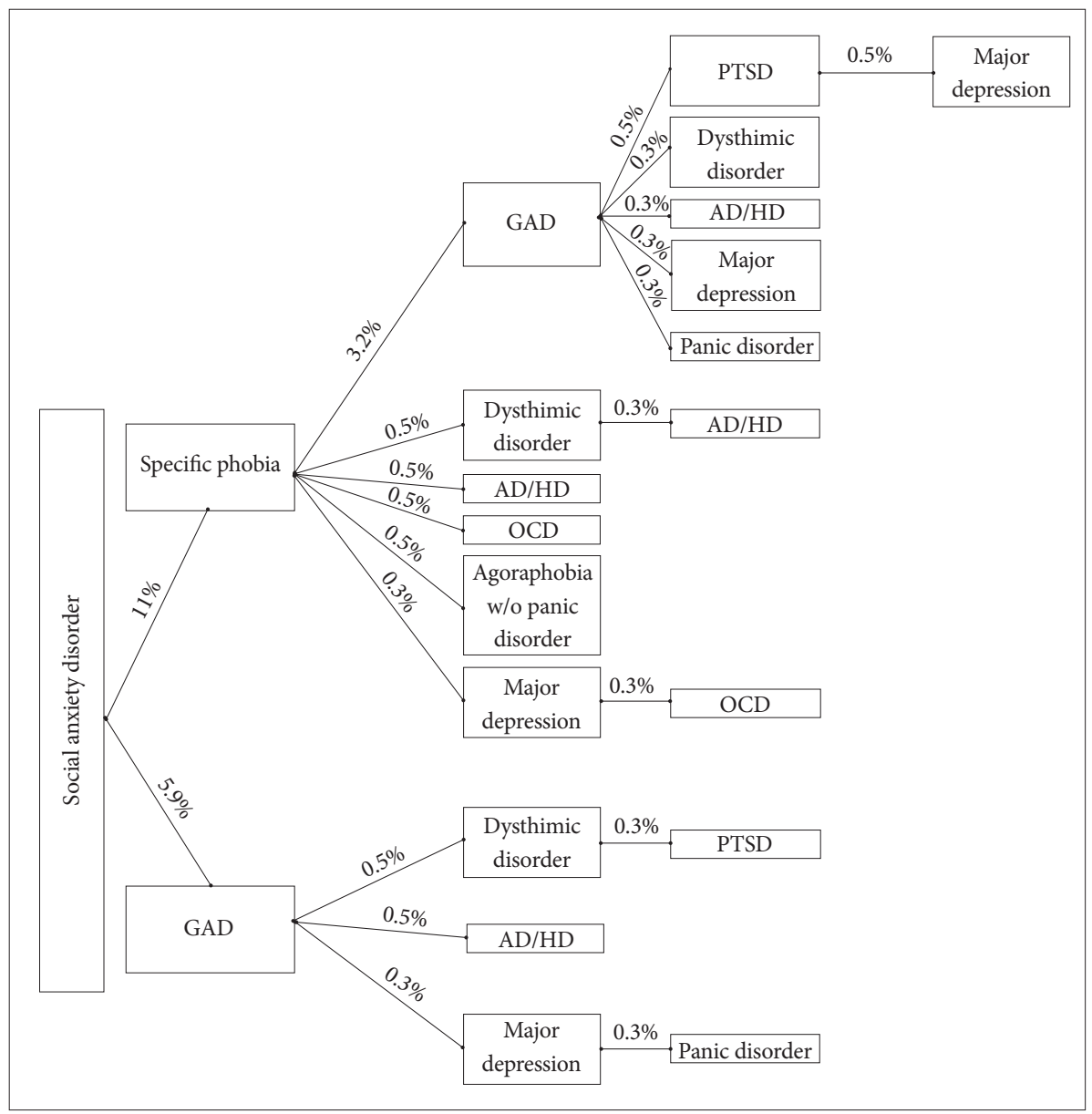

Figure 1. Percentages of comorbidity rates compared to total sample $(\mathrm{N}=409)$. GAD: generalized anxiety disorder, PTSD: posttraumatic stress disorder, AD/HD: attention-deficit and hyperactivity disorder, OCD: obsessive-compulsive disorder. 
two or more aggrupation subgroups among comorbid disorders to social anxiety disorders based on total sample $(n=409)$.

\section{DISCUSSION}

Comorbidity rates (33\%) of SAD were similar to previous treatment studies with adolescent samples. ${ }^{9}$ Unique data revealed that three anxiety disorders $(\mathrm{SAD}, \mathrm{GAD}$ and specific phobia) tend to be grouped together because of their high level of comorbidity. The data urges to mental health providers, pediatricians and school counselors to screen for GAD and specific phobia when assessing $\mathrm{SAD}$ in youth. In addition, major depression should be ruled-out when adolescents met criteria for GAD and other anxiety disorders (PTSD, OCD, PD) comorbid to SAD. Regarding to OCD, cases were evident when specific phobia was diagnosed, and unrelated to any other anxiety disorders, consistent with previous research. ${ }^{10}$ Unlike, comorbid PD and PTSD was diagnosed exclusively when GAD was present. Comorbid AD/HD and Dysthimic Disorder were mostly related to $\mathrm{GAD}$, in line with studies relationship between emotional disorders and AD/HD. ${ }^{11}$ Finally, comorbid agoraphobia with or without panic disorder had a unique comorbidity with specific phobia.

Clinicians should examine mood and other anxiety symptoms (e.g,. GAD) and differentiate them from SAD, which are typically and exclusively focused on interpersonal contexts, but may sometimes appear similar in their clinical presentation and co-occur with SAD. The high level of comorbidity between $\mathrm{SAD}$ and other disorders may be partially explained by substantial overlap of symptomatology assessed by DSMIV or common etiological factors (which might support transdiagnostic approaches). ${ }^{12}$ Further studies should examine coocurrence of SAD with DSM-5 criteria for other anxiety, mood and additional mental health disorders.

\section{REFERENCES}

1. American Psychiatric Association. Diagnostic and statistical manual of mental disorders, 5th Edition. Arlington: American Psychiatric Publishing; 2013.

2. Ranta M, La Greca AM, Garcia-Lopez LJ, Marttunen M. Introduction. In: Ranta M, La Greca AM, Garcia-Lopez LJ, Marttunen M, Editors. Social Anxiety and Phobia in Adolescents: Development, Manifestation and Intervention Strategies. Cham: Springer International Publishing; 2015, p. 1-7.

3. Knappe S, Sasagawa S, Creswell C. Developmental Epidemiology of Social Anxiety and Social Phobia in Adolescents. In: Ranta M, La Greca AM, Garcia-Lopez LJ, Marttunen M, Editors. Social Anxiety and Phobia in Adolescents: Development, Manifestation and Intervention Strategies. Cham: Springer International Publishing; 2015, p. 39-70.

4. Garcia-Lopez LJ, Ingles C, Garcia-Fernandez JM. Exploring the relevance of gender and age differences in the assessment of social fears in adolescence. Soc Behav Personal 2008;36:385-390.

5. Ingles C, Piqueras JA, Garcia-Fernandez JM., Garcia-Lopez LJ, Delgado D, Ruiz-Esteban C. Gender and age differences in the cognitive, psychophysiological, and behavioral responses of social anxiety in adolescence. Psicothema 2010;22:376-381.

6. Essau C, Conradt J, Petermann F. Frequency and comorbidity of social phobia and social fears in adolescents. Behav Res Ther 1999;37:831843.

7. Silverman WK, Albano AM. The Anxiety Disorders Interview Schedule for DSM-IV-Child and Parent Versions. London: Oxford University Press; 1996.

8. Garcia-Lopez LJ, De Los Reyes A, Salvador MC. Assessment of Social Anxiety in Adolescents. In: Ranta M, La Greca AM, Garcia-Lopez LJ, Marttunen M, Editors. Social Anxiety and Phobia in Adolescents: Development, Manifestation and Intervention Strategies. Cham: Springer International Publishing; 2015, p. 121-150.

9. McLellman LF, Alfano CA, Hudson JL. Cognition-Focused Interventions for Social Anxiety Disorder among Adolescents In: Ranta M, La Greca AM, Garcia-Lopez LJ, Marttunen M, Editors. Social Anxiety and Phobia in Adolescents: Development, Manifestation and Intervention Strategies. Cham: Springer International Publishing; 2015, p. 225250.

10. Melfsen S, Walitza S, Warnke A. The extent of social anxiety in combination with mental disorders. Eur Child Adolesc Psychiatry 2006;15: 111-117.

11. Huh Y, Choi I, Song M, Kim S, Hong SD, Joung Y. A comparison of comorbidity and psychological outcomes in children and adolescents with attention-deficit/hyperactivity disorder. Psychiatry Investig 2011; 8:95-101.

12. Garber J, Weersing R. Comorbidity of anxiety and depression in youth: Implications for treatment and prevention. Clin Psychol (New York) 2010;17:293-306. 